\title{
Characterization of PPACs' time resolution for fission studies
}

\author{
Diego Tarrío ${ }^{1, *}$, Alexander V. Prokofiev ${ }^{1}$, Keita Nakano $^{2}$, and Stephan Pomp ${ }^{1}$ \\ ${ }^{1}$ Uppsala University, Uppsala, Sweden \\ ${ }^{2}$ Kyushu University, Kyushu, Japan
}

\begin{abstract}
The existing Medley setup is being upgraded with Parallel Plate Avalanche Counters (PPACs) to measure neutron-induced fission cross sections. Single-gap PPACs have been developed for that purpose. The time resolution of the PPACs have been measured using a dedicated setup where, either $\alpha$ particles or fission fragments, are detected in coincidence by three PPACs and a Silicon detector. The results reported here demonstrate that the developed PPACs are suitable for the intended measurements in the white neutron beam at the NFS facility at GANIL.
\end{abstract}

\section{Introduction}

A Parallel Plate Avalanche Counter (PPAC) is a type of gas-filled detector widely used for detection of heavy ions [1]. A PPAC consists of two thin parallel electrodes separated by a gap filled with a gas at a few mbar pressure. In order to keep a high electric field and a good time resolution, the gap width is usually of a few mm.

Thanks to the low sensitivity to weakly ionizing particles, PPACs are suitable for detecting heavy ions (e.g. fission fragments) originating from neutron-induced reactions, in particular, at a neutron spallation source where they can be directly exposed to intense fluxes of neutrons and gamma rays.

PPACs will be used as a part of the upgraded Medley setup, which is briefly described in Section 2, with the aim of being able to measure neutron-induced fission cross sections at a neutron source, such as the Neutrons For Science (NFS) facility [2] at GANIL (France). Single-gap PPACs have been developed for that purpose. In the present paper, we report on the current status of the development of the PPACs for the mentioned setup and, in particular, we focus on recent measurements of the time resolution of these detectors. Further, the results of those characterization measurements serve as a starting point for a discussion on the suitability of the developed detectors for the intended purpose.

\section{The upgraded Medley setup}

One of the first Medley-based projects at NFS will focus on measurements of the cross sections and angular distributions of neutron-induced fission of ${ }^{235} \mathrm{U}$ and ${ }^{238} \mathrm{U}$ isotopes in the energy range $1-40 \mathrm{MeV}$. The key feature of the project is the simultaneous measurement of these two cross sections with respect to the neutron-proton elastic scattering, that is the most fundamental neutron standard.

\footnotetext{
*e-mail: diego.tarrio@physics.uu.se
}

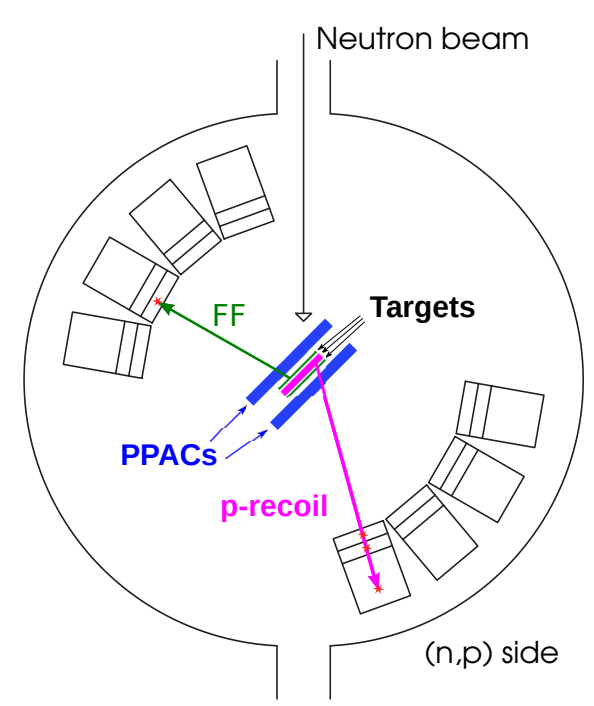

Figure 1. A layout of the upgraded Medley setup for (n,f) cross section measurements with respect to $n-p$ elastic scattering. The fission fragments will be detected by one of the PPACs and stopped in the first Si detector in one of the eight telescopes. The proton recoil from $\mathrm{H}(\mathrm{n}, \mathrm{p})$ will be stopped in the $\mathrm{CsI}(\mathrm{Tl})$ scintillator in one of the front telescopes, because of the kinematics of the reaction [4].

The original Medley (see Ref. [3] for an overview of earlier experiments) is a cylindrical vacuum chamber equipped with eight three-element telescopes, thus implementing $\Delta \mathrm{E}-\Delta \mathrm{E}-\mathrm{E}$ technique for particle identification. Each telescope consists of two fully depleted $\Delta E$ silicon surface barrier detectors (SSBD) of $50 \mu \mathrm{m}$ and $1 \mathrm{~mm}$ in thickness, respectively, and one $\mathrm{CsI}(\mathrm{Tl})$ scintillator crystal of $50 \mathrm{~mm}$ length, which suffices to fully stop recoil protons. The telescopes are mounted at $20^{\circ}$ intervals in two sets, covering the forward and backward hemispheres. 
The incident neutron energy is determined by measuring the neutron time of flight (TOF). The start signal of the TOF will be given by the accelerator. In case of lightion production measurements, the stop signal is given by detection of an ion in a telescope, with a subsequent correction for ion's TOF between the production target and the telescope. In case of fission measurements, the stop signal will be provided by the detection of a fission fragment $(\mathrm{FF})$ in a PPAC located close to the fission target. This is the only way to know the energy of the incoming neutron in a white neutron beam. This measurement is also necessary in a quasi-monoenergetic neutron beam, to discriminate between the neutrons in the high-energy peak and in the low-energy tail. A layout of the upgraded Medley setup can be seen in Fig. 1 [4].

\section{Description of the PPAC prototypes}

A PPAC consists of two parallel thin electrodes separated by a gap, a few mm wide, filled with a gas at low pressure. Ions (e.g. fission fragments) passing through the PPAC ionize the gas, thus releasing electrons. If the applied electric field between the electrodes exceeds a certain value (typically about hundreds $\mathrm{V} / \mathrm{cm} / \mathrm{mbar}$ ), those electrons will initiate avalanches of secondary ionizations. The swarm of electrodes will be collected by the anode, where an electrical signal is produced [1].

Several prototypes of PPACs have been developed and produced by the authors with the aim of being part of the upgraded Medley setup. The particular design of PPACs characterized in the present study comprises two thin parallel electrodes with an area of $160 \times 100 \mathrm{~mm}^{2}$ and separated by a $3.2 \mathrm{~mm}$ gap filled with $C_{3} F_{8}$ gas at a pressure of few mbar ( 7 mbar for the measurements reported here). Each electrode consists of a $0.9-\mu m$ thick mylar foil, on which a 40-nm thick layer of Aluminium has been deposited by evaporation. The bias voltage is applied to the anode, whereas the cathode is connected to the ground. The signal is read from the anode.

\section{Measurements of PPACs' time resolution}

\subsection{Experimental method}

The time resolution of the PPACs was measured using a setup with three PPACs, according to the layout shown in Fig. 2. A radioactive source (either ${ }^{241} \mathrm{Am}$ or ${ }^{252} \mathrm{Cf}$ ) was placed in one side, so that the particles emitted by the source would pass through three PPACs, and reach a silicon detector placed on the other side. The distance between the Si detector and the source amounted to $102 \mathrm{~mm}$ for ${ }^{241} \mathrm{Am}$, and $112 \mathrm{~mm}$ for ${ }^{252} \mathrm{Cf}$ (they use different sample holders). The Si detector was used as a trigger and also for selection of events caused by particles of interest ( $\alpha$ or FF) in a particular experiment. The smaller solid angle of the silicon detector, compared to the ones of the PPACs, ensured the selective detection of particles that arrived at the PPACs at close-to-normal incident angles, amounting to $0^{\circ}-6^{\circ}$ for $\mathrm{FF}$, and to $0^{\circ}-7^{\circ}$ for $\alpha$ particles. Each PPAC produced a signal, and we measured a difference between the times of arrival of those signals to the data acquisition system. This quantity, further referred to as "time difference", is defined by the TOF of the particle of interest between the two given PPACs, summed with a constant term due to unequal signal propagation times in the respective electronic circuits.

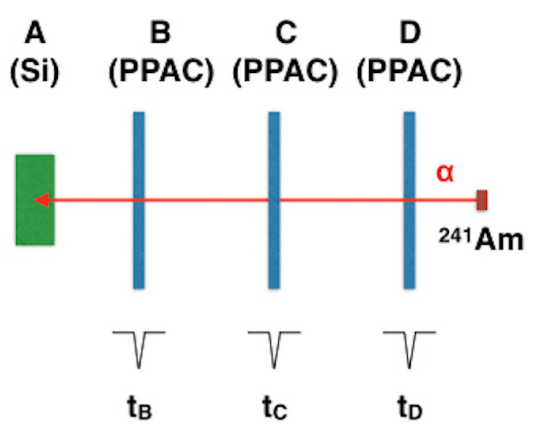

Figure 2. Setup for measuring the time resolution of the PPACs.

The measured time difference for a particle passing through any two of the three PPACs (identified as B, C, D, according to Fig. 2), has a distribution with a certain width $\left(\sigma_{B C}, \sigma_{B D}\right.$, or $\left.\sigma_{C D}\right)$ given by the following equations:

$$
\begin{gathered}
\sigma_{B C}^{2}=\sigma_{B}^{2}+\sigma_{C}^{2}+\sigma_{B C, \text { elect }}^{2}+\sigma_{B C, \text { energy }}^{2} \\
\sigma_{B D}^{2}=\sigma_{B}^{2}+\sigma_{D}^{2}+\sigma_{B D, \text { elect }}^{2}+\sigma_{B D, \text { energy }}^{2} \\
\sigma_{C D}^{2}=\sigma_{C}^{2}+\sigma_{D}^{2}+\sigma_{C D \text {,elect }}^{2}+\sigma_{C D, \text { energy }}^{2}
\end{gathered}
$$

where $\sigma_{B}, \sigma_{C}$, and $\sigma_{D}$ are the time resolutions of individual PPACs, the terms with the subindex "elect" describe contributions to the time resolution originating from the electronics associated to the given pair of PPACs, and the terms with the subindex "energy" are due to energy distributions of the incident particles. The latter term accounts for the fact that the energy distribution of the particles emitted by the source is not monoenergetic. Moreover, the energy losses in the gas and in the electrodes of the PPAC contribute to a further broadening of the energy distribution that translates into a certain contribution to the spread in the measured time difference. This contributions are more pronounced for the case of the propagation of FF.

The contributions of the electronics to the measured time resolutions, $\sigma_{B C \text {,elect }}, \sigma_{B D \text {,elect }}$, and $\sigma_{C D \text {,elect }}$ were determined using a pulse generator producing two identical signals that were sent to the electronics along the same circuits that were used for the PPAC signals. The distribution of time differences was measured in the same way as with the PPACs. Because of the different gains in the amplifier used for $\alpha$ particles and for FF, this determination has been performed separately for each of the setups.

Thanks to the fact that each particle passes through 3 PPACs, we have a system of 3 equations with 3 unknowns, so that it is possible to obtain the individual time contributions $\sigma_{B}, \sigma_{C}, \sigma_{D}$ from each detector, following the procedure used in [5]. The time resolution is defined as the full 


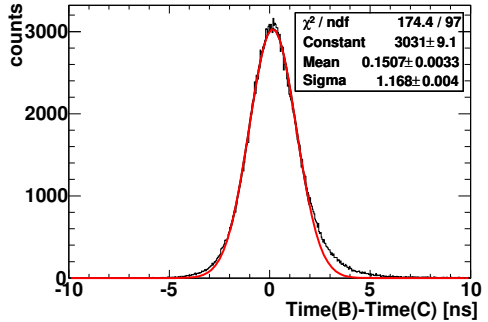

(a) PPACs B and C

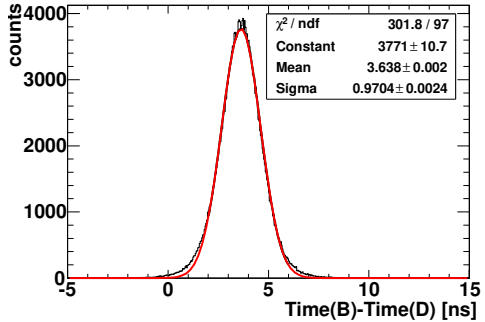

(b) PPACs B and D

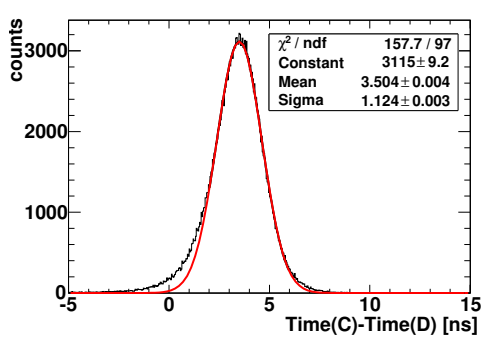

(c) PPACs C and D

Figure 3. Measured time-difference distributions (in black) and Gaussian fits (in red) for the case of $\alpha$ particles from ${ }^{241} \mathrm{Am}$. Each plot represents a different pair of PPACs.

width at half maximum (FWHM) of the distribution, that is directly related to $\sigma$ by: $F W H M=2 \sqrt{2 \cdot \ln 2} \cdot \sigma$.

\subsection{Results for $\alpha$ particles}

Alpha particles from a source of ${ }^{241} \mathrm{Am}$ were used in one of the setups. The energy spectrum of the emitted $\alpha$ particles has a peak at $\sim 5.49 \mathrm{MeV}$ with the FWHM of $16 \mathrm{keV}$, as reported by the manufacturer of the source [6]. Such a spread in energy can only cause a negligible contribution ( $\sim 8 \mathrm{ps)}$ to the time spread observed with the last PPAC (B), so that the $\alpha$ source can be regarded as monoenergetic. Therefore, the straggling observed in the kinetic energy (and velocity) of the $\alpha$ particles along the setup is only due to the energy losses in the PPAC foils and in the low-pressure gas that fills the reaction chamber. A simulation with Geant4 [7] has been done to determine the energy distribution of the $\alpha$ particles arriving to each of the detectors. Further, using kinematics calculations, it was found that the spread in energy corresponds to the following contributions to the time spreads: $\sigma_{B C \text {,energy }}=0.017$ $\mathrm{ns}, \sigma_{C D \text {,energy }}=0.008 \mathrm{~ns}$, and $\sigma_{B D \text {,energy }}=0.015 \mathrm{~ns}$, which are negligible compared to the contribution from the electronics (in the order of $1 \mathrm{~ns}$, for the $\alpha$ setup).

The experimental results of the time-difference distributions for the three possible pairs of the PPACs are shown in Fig. 3. Gaussian fits are also shown, from where the values of $\sigma$ are deduced. By solving the system of equations (1), the time resolution of the PPACs, defined by the FWHM, are 1.08, 1.75, and $0.84 \mathrm{ns,} \mathrm{for} \mathrm{PPACs} \mathrm{B,} \mathrm{C} \mathrm{and}$ $\mathrm{D}$, respectively. The uncertainties in the measured resolutions do not exceed $0.02 \mathrm{~ns}$.

\subsection{Results for fission fragments}

Since the ultimate purpose of the developed PPACs is to detect FF in fission cross-section measurements, the time resolution of PPACs was also investigated using fragments from the decay of ${ }^{252} \mathrm{Cf}$. FF are not at all monoenergetic and follow a certain distribution of masses and kinetic energies. A similar procedure to the one outlined in the previous subsection was followed with the ${ }^{252} \mathrm{Cf}$ source; contrary to the case of $\alpha$ 's from ${ }^{241} \mathrm{Am}$, the spread in velocity of the different fragments contributes in a significant way to the measured width of the time-difference distributions.
Moreover, the ${ }^{252} \mathrm{Cf}$ source used in this experiment is covered by a $1 \mathrm{mg} / \mathrm{cm}^{2}$ Nickel foil, what contributes significantly to the broadening of the velocity distribution.

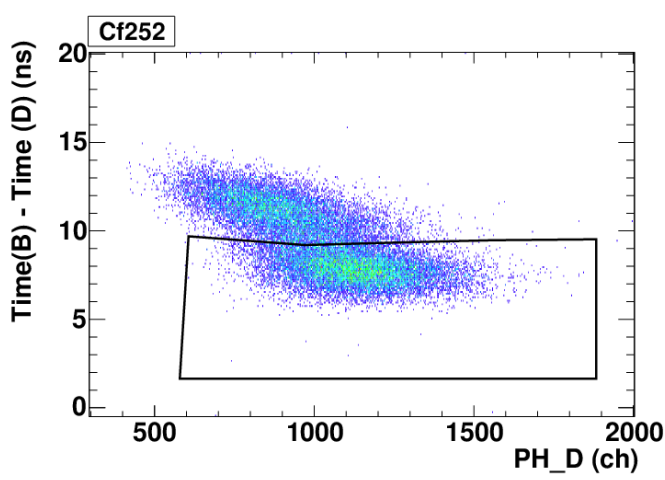

Figure 4. Time difference for FF between PPACs B and D versus the pulse height of the signal in PPAC D. The black contour selects the light fission fragments.

The experimental measurement of the time difference between PPACs B and D (the most distant ones) allows to distinguish between the heavy (HFF) and light fission fragments (LFF) thanks to their different velocities. A plot of time differences versus the amplitude of the signal in PPAC D makes this separation clearer and makes also possible to select events corresponding to LFF, as shown in Fig. 4 by the black contour. By selecting those events, the three possible time distributions between PPACs can be obtained for the LFF (as it was done for the $\alpha$ particles).

The energy losses along the setup, included those in the $\mathrm{Ni}$ foil, were simulated with Geant4. As an input for the event generator in Geant4, we used fission fragment distributions for the ${ }^{252} \mathrm{Cf}$ decay obtained with the GEF code [8]. According to the calculations, the energy losses along the setup contribute to the spread in the arrival time of LFF at the PPACs by $0.09,0.26$, and $0.46 \mathrm{~ns}$ for the PPACs D, C, and B, respectively. The measured timedifference distributions and respective gaussian fits, for the three pairs of PPACs, are shown in Fig. 5.

The contribution of the electronics to the time spread was measured and found to be $0.4 \mathrm{~ns}$ in all the three cases. 


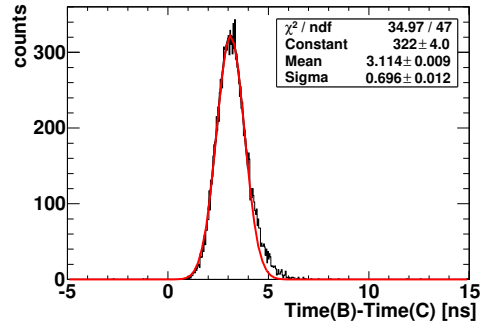

(a) PPACs B and C

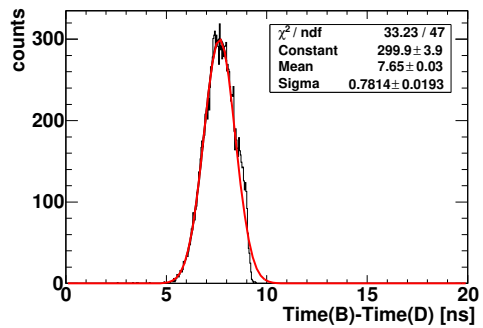

(b) PPACs B and D

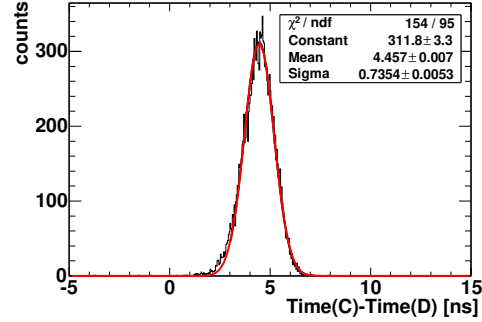

(c) PPACs C and D

Figure 5. Measured time-difference distributions (in black) and Gaussian fits (in red) for the case of light fission fragments from ${ }^{252} \mathrm{Cf}$. Each plot represents a different pair of PPACs.

The different value with respect to the case of the $\alpha$ particles is because of different gains in the amplifiers.

By solving the system of equations (1), the individual values for the time resolution of PPACs B, C, and D, are found to be $0.8,1.1$, and $0.7 \mathrm{~ns}$, respectively. The uncertainties do not exceed $0.2 \mathrm{~ns}$.

\subsection{A comparison of PPAC time resolution for $\alpha$ particles and FF}

In Table 1 we present the measured time resolutions of the different PPACs, for the purpose of comparison between the cases of FF- and alpha particle detection.

\begin{tabular}{|c|c|c|}
\hline \multicolumn{3}{|c|}{ Time resolution, FWHM [ns] } \\
\hline PPAC & $\alpha$ particles & Light Fission Fragments \\
\hline B & $1.08 \pm 0.02$ & $0.8 \pm 0.2$ \\
C & $1.75 \pm 0.02$ & $1.1 \pm 0.2$ \\
D & $0.84 \pm 0.02$ & $0.7 \pm 0.2$ \\
\hline
\end{tabular}

Table 1. Results for the time resolution of the different PPACs with $\alpha$ particles and light fission fragments.

As seen in the table, all tested PPACs have shown better time resolution for FF than for $\alpha$ particles. This conclusion remains valid even if we do not take into account the term describing the contribution from energy and mass distribution of the fragments. Consequently, the treatment of FF distributions is not responsible for the difference between the results for FF and $\alpha$ particles. We interpret this finding as a manifestation of the more intense ionization produced in the PPACs by the FF compared to $\alpha$ particles.

\subsection{Implications of the results for fission studies with Medley at NFS}

The closest position for the sample target at NFS will be at approximately 5.5 meters from the neutron production target. Consequently, if the neutron TOF is measured with an uncertainty of $1 \mathrm{~ns}$ (that is the typical time resolution obtained here for the PPACs), the uncertainty in the neutron energy will be of $3.3 \%$ at $40 \mathrm{MeV}$, that is the maximum achievable energy of the facility. At $1 \mathrm{MeV}$, where several actinides have their fission threshold, the uncertainty would be of $0.5 \%$, or $0.005 \mathrm{MeV}$.
That resolution is sufficient for identifying the existing vibrational resonances exhibited by several actinides around the fission threshold (such as ${ }^{234} \mathrm{U},{ }^{238} \mathrm{U}$, or ${ }^{232} \mathrm{Th}$, for example), required to model their fission barriers [9].

Further development in the electronics readout is being done with the aim of improving the present values of the time resolution. If succeed, the neutron energy will be measured with a better resolution. A detailed analysis on all the contributions to the measured neutron energy, including those related to the facility, was in Ref. [10].

\section{Conclusions}

Several PPAC detectors have been developed and built as a part of the upgrade of the Medley setup, aiming at future measurements of neutron-induced fission cross sections.

A method to measure the individual time resolution of each PPAC has been presented, as well as the results obtained using $\alpha$ particles from ${ }^{241} \mathrm{Am}$ and $\mathrm{FF}$ from ${ }^{252} \mathrm{Cf}$.

It was found a better time resolution for fission fragments than for $\alpha$ 's, most likely due to their higher ionization that produce larger signals in the detectors.

The values obtained for the time resolution are adequate for the use of the setup in a white neutron spectrum and, in particular, to study fission cross sections. Furthermore, it is expected that the time resolution of the electronics can still be further optimized with the use of faster amplifiers, what would improve the achievable resolution in the measured energy of the incident neutrons in fission cross section measurements.

\section{References}

[1] F. Sauli, Gaseous Radiation Detectors (Cambridge University Press, United Kingdom, 2014) 160-181

[2] X. Ledoux et al., EPJ Web Of Conf. 146, 03003 (2017)

[3] S. Pomp et al., EPJ Web Of Conf. 8, 07013 (2010)

[4] D. Tarrío et al., EPJ Web Of Conf. 146, 03026 (2017)

[5] C. Paradela et al., Nucl. Inst. Meth. A 735, 94 (2014)

[6] L. Einarsson, private communication (2016)

[7] S. Agostinelli et al., Nucl. Inst. Meth. A 506, 250 (2003)

[8] K. H. Schmidt et al, Nucl. Data Sheets 131, 107 (2016)

[9] M. Sin et al., Phys. Rev. C 74, 014608 (2006) 